\title{
Cross-cultural psychometric assessment of the parent-teen sexual risk communication (PTSRC-III) scale in Jamaica
}

\author{
Norman K. Waldron ${ }^{1 *}$, M. Katherine Hutchinson ${ }^{2}$, Hermi H. Hewitt ${ }^{3}$, Eulalia Kahwa ${ }^{3}$, \\ Pansy I. Hamilton ${ }^{4}$ \\ ${ }^{1}$ Department of Community Health and Psychiatry, The University of the West Indies (UWI), Kingston, Jamaica; \\ *Corresponding Author: norman.waldron@uwimona.edu.jm \\ ${ }^{2}$ New York University College of Nursing, New York, USA \\ ${ }^{3}$ The UWI School of Nursing, The University of the West Indies (UWI), Kingston, Jamaica \\ ${ }^{4}$ Hugh Wynter Fertility Management Unit, Faculty of Medical Sciences, The University of the West Indies (UWI), Kingston, Jamaica
}

Received 5 January 2012; revised 18 February 2012; accepted 11 March 2012

\section{ABSTRACT}

Background: HIVIAIDS represents a serious health threat in Jamaica. Adolescent and young adult females are at particularly high risk. US studies have shown that parent-teen sexual communication may significantly influence the sexual riskrelated beliefs and behaviors. However, no studies have examined parent-teen sexual communication in Jamaica or the wider Caribbean. Objective: The study aims to describe patterns of parent-teen sexual communication between Jamaican mothers and adolescent daughters and assess the psychometric properties of the Parent-Teen Sexual Risk Communication (PTSRC-III) scale when used with this population. Method: Data were collected as part of a larger randomized controlled trial study. A total of 330 urban Jamaican adolescent girls, ages 13 - 17, and their mothers or female guardians completed the 8-item PTSRC-III scale at baseline and 3month follow-up. Results: Mothers' and daughters' scores covered the full range of possible scores. Internal reliability of the PTSRC-III scale was excellent ( $\alpha=0.92$ and 0.93 for mothers and daughters, respectively). There was evidence of consistency over time $(r=0.57, p<0.001)$ and moderate mother-daughter concordance in reports of sexual communication $(r=0.27, p<$ 0.001). Daughters' PTSRC-III scores were significantly correlated with daughters' reports of comfort and ease with mother-daughter sexual communication $(r=0.427, p<0.001)$, motherdaughter closeness $(r=0.288, p<0.001)$, and importance of mothers' approval $(r=0.146, p=$ 0.008). A two-factor structure was identified, al- though the second factor showed a lower eigenvalue than was found in previous US studies. The loading pattern and explanation of variance were very similar to the factor 2 described in the initial psychometric testing of the PTSRC-III instrument. Discussion: Patterns of sexual communication and the psychometrics of the scale when used in Jamaica were consistent with US results, and provided evidence of the cross-cultural reliability and validity of the PTSRC-III scale.

Keywords: Adolescent Sexual Risk; Sexual Communication; Psychometric Assessment; Reliability

\section{INTRODUCTION}

In the Caribbean, HIV/AIDS rates are high, with only countries in sub-Saharan Africa having higher rates [1]. Adult HIV prevalence in the Caribbean ranges from a low of $0.1 \%$ in Cuba to a high of $3.1 \%$ in the Bahamas [1]. In Jamaica, the largest English-speaking Caribbean island, the HIV prevalence among adults, 15- to 49-yearold, is estimated to be $1.7 \%$ [1]. There are an estimated 32,000 (range 21,000 - 45,000) persons living with HIV/ AIDS in Jamaica. HIV/AIDS rates vary across the island, but are highest in urban areas (e.g., Kingston/St. Andrew and St. Catherine) and coastal tourist areas (St. Ann and St. James). In 2009, 66\% of reported HIV/AIDS cases were from these parishes [2].

The primary mode of HIV transmission in Jamaica is heterosexual contact, with more than $90 \%$ of adult cases attributed to heterosexual practices [2]. There is a great sex-disparity in HIV prevalence in adolescents and young adults in the Caribbean. HIV prevalence among 15- to 
24-year-old females in the Caribbean is twice that of males, $0.8 \%$ vs. $0.4 \%$, respectively [1]. These same disparities are seen in Jamaica [2-4].

\subsection{HIVIAIDS in Jamaican Adolescent Girls}

Jamaican female adolescents face much higher risk for HIV infection compared to male adolescents [3-6]. The cumulative AIDS case rate (per 100,000 population) among 10- to 19-year-olds was more than three times higher in females (62.7) compared to males (19.2) [2]. Several factors have been advanced to explain this elevated HIV/AIDS risk, including early sexual debut, unprotected sexual intercourse, sexual abuse, transactional sex with older men, and multiple sexual partnerships [5, 7-11]. More than half of Jamaican adolescent girls have sex before the age of 15 years [4-5,12-15]. Many adolescent girls engage in sexual relationships with older men in exchange for money or gifts $[6,12,13,16,17]$. Both early sexual debut and older male partners increase the likelihood of sexually transmitted infections (STIs) as well as HIV and unplanned pregnancy $[12,16,18]$. Often these girls lack condom use self-efficacy and/or have less power to refuse unsafe sex or negotiate condom use with older partners $[6,16]$.

Sexual coercion and intimate partner violence are also serious issues in Jamaica that may compromise young women's abilities to refuse sex or negotiate for condom use. Approximately $11.9 \%$ of sexually active Jamaicans, 15- to 49-years-old, report they have been coerced or pressured to have sex. The proportion is even higher (13.1\%) among adolescent and young adult women, 15to 24-years-old [16]. Sexual coercion and partner violence are more likely to occur among adolescent girls who are younger than their male sexual partners $[4,12$, 14,15,17-19].

Jamaican adolescent girls and young women who engage in transactional sex, casual sex and sex with multiple partners use condoms less consistently than others $[6,12,14-16]$. Further complicating their risk is the lack of perceived risk for HIV and other sexually transmitted infections (STIs). Almost two-thirds of Jamaicans who report having multiple sexual partners and using condoms infrequently believe that they had little or no risk of contracting HIV infection [16].

\subsection{Parent Influences of Adolescent Sexual Risk}

Interactions between parents/caregivers and their adolescent children have been shown to substantially influence the sexual risk beliefs and behavior of adolescents. US studies have identified a number of important parental influences, including parental monitoring and supervision, high quality parent-teen relationships, parent-teen sexual communication and less permissive parental attitudes towards adolescent sex [20-34]. Of these, parentchild sexual communication has been singled out as being particularly important. Parent-teen sexual communication has been associated with improved adolescent resistance to sexual pressure from peers, delays in sexual initiation, and reduced sexual risk-taking [26-32,35-39]. Unfortunately, there is a dearth of research outside of the US examining family influences of adolescent sexual risk taking. Although a few studies have been undertaken in Europe [40] and Mexico [37,38], no such studies have been conducted in the Caribbean.

\subsection{Parental Expansion of the Theory of Planned Behavior}

Consistent with the findings in the literature noted above, the Parental Expansion of the Theory of Planned Behavior [35] provides a conceptual framework to guide the study of family influences on adolescent sexual risk. The original Theory of Planned Behavior (TPB) [41] is a widely used model for understanding individuals' intentions to perform a given behavior. In short, the TPB posits that individuals' intentions are determined by their attitudes and beliefs toward the behavior, their perceptions of whether important others would approve, and their perceived control over the behavior. The Parental Expansion of the TPB builds upon the original model by explicitly acknowledging that parents are among the most proximal and important influences of adolescents' sexual risk-related beliefs and, in turn, their intentions and behaviors [35]. In both the TPB and the Parental Expansion of the TPB, there is a recognition that beliefs, intentions and behaviors occur within and are influenced by the larger socio-cultural context. In addition, the relative importance of behavioral beliefs, normative beliefs, and control beliefs may vary from culture to culture. Thus, when using either model to understand behavior or develop behavioral interventions, extensive elicitation research is needed to understand the culture-specific influences operating within a given group $[35,42,43]$. Culturally sensitive and valid instruments are also needed to assess influential parenting behaviors and other salient influences of adolescent sexual risk behavior.

\subsection{The Parent-Teen Sexual Risk Communication Scale}

The Parent Teen Sexual Risk Communication Scale (PTSRC) was developed to quantify and measure one of the most important parental influences of adolescent sexual beliefs and behaviors, sexual communication. The scale assesses the amount of informational sexual communication occurring between adolescents and each of their parents or guardians [24]. The most recent version, 
the PTSRC-III, has undergone extensive psychometric testing and demonstrated a stable two factor structure, reliability and validity when used with various adolescent groups within the US (e.g., college students, late adolescent licensed drivers, adolescent females) [24,31]. In addition, the scale has been employed in studies in Mexico $[37,38]$, and in ongoing studies in South Africa and Jamaica.

However, the cross-cultural validity of instruments may not be assumed. A cross-cultural assessment of the PTSRC-III is important to determine whether the scale is a valid and reliable measure of parent-teen sexual risk communication when used with Jamaican families, and to assess whether the instrument has construct and functional equivalence and is free of item or construct bias $[44,45]$. To the best of our knowledge, there have been no studies examining parental influences of adolescent sexual risk and no known measures of parent-child sexual communication validated for use in the Caribbean. Thus, the aims of this study were to: 1 ) describe patterns of mother-daughter sexual risk communication between urban Jamaican adolescent girls, ages 13 - 17, and their mothers; and 2) examine the psychometric properties of the PTSRC-III scale when used with urban Jamaican families.

\section{METHODS}

\subsection{Study Design}

The data were collected as part of a larger, randomized controlled trial study evaluating a theory-based, culturespecific HIV risk-reduction intervention for Jamaican adolescent girls, ages 13 - 17, and their mothers. Mother and daughter dyads were recruited into the trial and randomly assigned to participate in either an HIV risk-reduction intervention or a general health promotion intervention. The two groups were equivalent; there were no baseline differences between the control and intervention groups on demographic characteristics and main study variables [46].

All instruments and data collection procedures were pilot tested prior to their use in the main study. The 8-item PTSRC-III scale was included on the mothers' and daughters' pre-intervention baseline, 3-month follow-up and 6-month follow-up questionnaires. Baseline and 3-month follow-up data are reported here.

\subsection{Procedure}

The project was reviewed and approved by the New York University (NYU) University Committee on Activities Involving Human Subjects (UCAIHS) and the Ethics Committee of the Faculty of Medical Sciences, University of the West Indies (FMS/UWI) prior to par- ticipant recruitment and data collection. Prior to participation, mothers provided signed written informed consent for themselves and for their daughters, while daughters provided signed written informed assent in a separate room. After obtaining informed consent, data were collected by trained data collectors via paper-and-pencil questionnaires; because of literacy concerns, the questionnaires were also projected on the front wall of the room and read aloud during data collection. In addition, individual reading assistance was available and provided as needed in a discrete manner.

\subsection{Sample}

The sample consisted of 330 mother-daughter dyads from Kingston, St. Andrew and St. Catherine, Jamaica (urban parishes located on the southern coast of the island). Participants were recruited through community based organizations and via word-of-mouth referrals. In order to participate, daughters had to be between the ages of 13 and 17, unmarried, not currently pregnant, reside in one of the three parishes, and self-identify as Jamaican. Mothers had to be the primary female caregiver of the adolescent participant. In order to be eligible, both the mother and the daughter had to agree to participate.

\subsection{Measures}

The key outcome measure, the Parent-Teen Sexual Risk Communication (PTSRC-III) scale, consisted of 8 Likert-type items that asked about the amount of communication that occurred in each of eight sexual topic areas (birth control, sexually transmitted infections [STIs], preventing STIs, preventing HIV/AIDS, condom use, pressure to have sex, how to resist sexual pressure, postponing sex). Both mothers and daughters completed the PTSRC-III scale at each data collection point. Item wording was slightly different as appropriate for mothers (e.g., "How much have you told your daughter about...”) or daughters (e.g., "How much has your mother told you about...”). Response choices ranged from 1 - 5 (nothing [1], very little [2], some [3], a lot [4], and everything [5]). Possible scores ranged from 8 - 40 with higher scores representing more extensive sexual risk communication between the mother/ mother figure and daughter [24].

Other measures included daughters' reports of age (in years), self-identified race/ethnicity, current school enrollment, living arrangements during the past year, condom use self-efficacy, and intentions to have sex in the next 3 months. Daughters also reported on their parents' marital status, the type of relationship with the mother/ mother figure/female guardian (e.g., biological mother, grandmother, aunt, etc.), perceived closeness to mother, perceived importance of mothers' opinions, and perceptions of mothers' attitudes toward teen sex with a steady 
boyfriend. Measures assessed by mothers' reports included mothers' age (in years), education level, and current employment status.

\subsection{Data Analysis}

All analyses were conducted using Statistical Package for Social Sciences (SPSS) PASW version 18. Associations between continuous variables were assessed using Pearson's product-moment coefficients $(r)$, while those between categorical variables were calculated using Spearman's rho $\left(r_{s}\right)$. All analyses utilized baseline data except where otherwise noted. Analyses that included data from the 3-month follow-up were limited to participants from the control group $(n=165)$ in order to avoid the potential confounding effects from participation in the HIV risk-reduction intervention. Although this decision reduced analytic power, the potential confound was seen as significant given that a major emphasis of the mothers' HIV risk-reduction intervention curriculum was on increasing mother-daughter sexual communication. T-tests for differences in mean scores were conducted using dependent t-tests as mothers and daughters were not independent participants and comparisons of baseline and 3-month follow-up scores constituted repeated measures.

\section{RESULTS}

\subsection{Participants}

As is summarized in Table 1, daughters ranged in age from 13 and 17 years, with a mean age of 14.7 (1.3) years $(n=330)$. Almost all (97.3\%) were currently enrolled in school. All self-identified as Jamaican (an inclusion criteria for the study). Most of the daughters (88.7\%) reported living with the mother/mother figure all or most of the time; nearly $82 \%$ reporting being close or very close to their mother/mother figure. The mothers' mean age was 39.8 (7.1) years $(n=330)$. Fewer than $15 \%$ of mothers were currently married to their daughter's father; approximately $1 / 3$ of mothers were employed full-time, while more than $1 / 3$ were unemployed. The participant retention rate at 3-month follow-up was $96.5 \%$ [46].

\subsection{PTSRC III Scale Scores}

The Daughters' baseline scores on the PTSRC-III scale extended across the full range of possible scores, 8 - 40, with a mean of 25.66 (9.14). As is shown in Table 2 , scores varied across items. Daughters reported the greatest amount of sexual communication with mothers for Item 8 (communication about waiting to have sex) (mean $=4.02$ [1.18]). In comparison, daughters reported the least amount of communication for Item 3 (com-
Table 1. Demographic characteristics of the sample.

\begin{tabular}{|c|c|}
\hline Demographic characteristic & Frequency \\
\hline Daughter's mean age in years $(S D)$ & $14.7(1.3)$ \\
\hline Daughters currently in school & 97.3 \\
\hline \multicolumn{2}{|l|}{ Self-identified race/ethnicity included: } \\
\hline Asian/Chinese ${ }^{\mathrm{b}}$ & 4.0 \\
\hline Hispanic & 2.1 \\
\hline East Indian & 11.3 \\
\hline Parents currently married & 14.7 \\
\hline \multicolumn{2}{|l|}{ Mother's ${ }^{c}$ relationship to daughter: } \\
\hline Biological mother & 89.5 \\
\hline Aunt & 4.9 \\
\hline Grandmother & 2.2 \\
\hline Other & 3.3 \\
\hline Lives with mother all or most of time ${ }^{a}$ & 88.7 \\
\hline Close or very close to mother ${ }^{\mathrm{a}}$ & 81.8 \\
\hline Mother's mean age in years $(S D)$ & $39.8(7.1)$ \\
\hline \multicolumn{2}{|l|}{ Mother’s education: } \\
\hline Primary school or less & 20.0 \\
\hline High school or vocational & 74.8 \\
\hline Tertiary or more & 5.2 \\
\hline \multicolumn{2}{|l|}{ Mother employed : } \\
\hline Full-time & 33.4 \\
\hline Part-time & 30.0 \\
\hline
\end{tabular}

Note: $N=330$. All frequencies are percentages unless otherwise noted as means $(S D)$. "in the past year; "'Chinese" is commonly used in Jamaica to denote "Asian"; “"Mother" referred to primary female caregiver/mother figure.

Table 2. PTSRC-III scale: mean item and total scores (baseline).

\begin{tabular}{lcc}
\hline \multicolumn{1}{c}{ PTSRC III Items } & Daughters & Mothers \\
\hline 1) Birth control & $3.02(1.47)$ & $3.25(1.41)$ \\
2) STIs & $3.12(1.39)$ & $3.72(1.18)$ \\
3) Preventing STIs & $2.85(1.39)$ & $3.74(1.21)$ \\
4) Prevent HIV/AIDS & $3.11(1.40)$ & $3.54(1.26)$ \\
5) Condom use & $2.82(1.44)$ & $3.02(1.43)$ \\
6) Pressure to have sex & $3.45(1.41)$ & $4.09(1.10)$ \\
7) How to resist sexual pressure & $3.28(1.47)$ & $3.98(1.12)$ \\
8) Postponing sex & $4.02(1.18)$ & $4.31(0.94)$ \\
Total Mean Score & $25.66(9.14)$ & $29.64(7.74)$ \\
\hline
\end{tabular}

Note: $N=330$. 
munication about preventing STIs) and Item 5 (communication about condoms) (means $=2.85$ [1.39] and 2.82 [1.44], respectively).

Jamaican mothers' baseline PTSRC-III scale scores also ranged from 8 - 40. Mothers reported slightly greater amounts of sexual communication, with a mean of 29.64 (7.74). Variation in mothers' individual item scores was nearly identical to that for daughters. Mothers' highest scores were for Item 8 (communication about waiting to have sex; mean $=4.31$ [0.94]) and lowest scores were for Item 5 (communication about condoms; mean $=3.02[1.43]$ ). In contrast to daughters, mothers also reported low scores for Item 1 (communication about birth control; mean = 3.25 [1.14]).

Mean PTSRC-III scores at the 3-month follow-up were similar to those at baseline. The mean score for daughters was 26.47 (9.48). The mean score for mothers at 3-month follow-up was 31.98 (6.88). Sample sizes for these analyses were 160 and 165, respectively.

In terms of congruency in mothers' and daughters' reports of sexual communication, baseline reports showed moderate levels of agreement $(r=0.27, p<0.001, n=$ 330 ), as did reports at the 3-month follow-up ( $r=0.22, p$ $<0.001, n=160)$. Mother-daughter differences in the baseline mean PTSRC-III scores were statistically significant $(t=7.07, d f=329, p<0.001)$. Similarly, at the 3-month follow-up ( $n=160)$, mothers' PTSRC-III mean scores were significantly $(t=6.77, d f=159, p<0.001)$ greater than those of their daughters.

\subsection{Reliability}

Cronbach's alpha was computed to estimate the internal reliability of the 8-item PTSRC-III scale. Cronbach's alpha coefficients for the daughters' and mothers' baseline scores were 0.93 and 0.92, respectively. Both Cronbach's alphas were above the minimal acceptable value of 0.80 for well-designed scales $[47,48]$. These high Cronbach's alphas indicated excellent internal consistency of the items when used with both Jamaican female adolescents and mothers. The inter-item correlation matrices showed that all items had positive and strong correlations, with correlation coefficients ranging between $0.51-0.80$.

In addition to internal reliability, the stability or testretest reliability of PTSRC scores was assessed by examining the consistency of PTSRC-III scale scores between baseline and 3-month follow-up. As was previously discussed, these analyses were limited to data from control group participants in order to avoid confounding effects that participation in the mother-daughter HIV risk-reduction intervention might have on motherdaughter sexual risk communication. Daughters' mean PTSRC-III scores at baseline and 3-month follow-up were 26.16 (8.69) and 26.47 (9.48), respectively. The two sets of scores were significantly correlated $(r=0.57$, $p<0.001, n=160)$; differences between the scores were not statistically significant ( $t=0.47, d f=159, p=0.64)$. Mothers' PTSRC-III scores increased between baseline (29.96 [7.11] and 3-month follow-up (31.98 [6.88]). Mothers' scores at the two time points were significantly correlated ( $r=0.70, p<0.001, n=165$ ); the differences between the two sets of mothers' scores were significant $(t=4.74, d f=164, p<0.001)$.

\subsection{Factor Analysis}

As is shown in Table 3, factor analysis for the 8-item PTSRC-III scale (using daughters' reports) was undertaken using principal component analysis with confirmatory factor analysis [49]. The scree-plot, which shows eigenvalues in decreasing order, revealed one factor having an eigenvalue greater than 1 , and a second factor with an eigenvalue just under 1 . Factor 1, termed "Communication related to management or reduction of risk for adverse sexual outcomes” [24], included items 1, 2, 3, 4 and 5 and had an eigenvalue of 5.382. Factor 1 accounted for $67.3 \%$ of the variance. The only other meaningful factor included items 6, 7 and 8. This factor was previously labeled "Communication related to the prevention of sexual risk through prevention of sexual activity" [24]. This second factor had an eigenvalue of 0.626 and explained an additional $7.8 \%$ of the variance. The two factors were highly correlated ( $r=0.717, p<$ $0.001)$; together they explained $75.1 \%$ of the variance.

\subsection{Validity}

Evidence of concurrent validity was found by examining the associations between daughters' PTSRC-III scores and other variables that, based on the published literature, one would expect to be related to parent-teen sexual communication. As is shown in Table 4, daughters' reports of PTSRC were significantly associated with

Table 3. Pattern matrix-principal component analysis.

\begin{tabular}{lcc}
\hline \multicolumn{1}{c}{ PTSRC-III Items } & Factor 1 & Factor 2 \\
\hline 1) Birth control & 0.798 & 0.030 \\
2) STIs & 0.785 & 0.114 \\
3) Preventing STIs & 0.926 & -0.056 \\
4) Preventing HIV/AIDS & 0.833 & 0.103 \\
5) Condom use & 0.873 & -0.059 \\
6) Pressure to have sex & 0.305 & 0.599 \\
7) How to resist sexual pressure & 0.141 & 0.770 \\
8) Postponing sex & -0.101 & 0.959 \\
\hline
\end{tabular}

Note. $N=330$. Rotation Method: Oblimin with Kaiser normalization, SPSS (version 18). 
Table 4. Bi-variate correlations between daughters' reports of PTSRC and other variables.

\begin{tabular}{lcc}
\hline \multicolumn{1}{c}{ Variable } & Correlation & $p$ \\
\hline Mothers' reports of PTSRC ${ }^{\mathrm{a}}$ & 0.272 & $<0.001$ \\
Daughters' age & 0.074 & 0.178 \\
Comfortable talking with mother about sexual topics $\left(r_{s}\right)$ & 0.427 & $<0.001$ \\
How easy would be to talk to mother about sex $\left(r_{s}\right)$ & 0.365 & $<0.001$ \\
Mother-daughter closeness & 0.288 & $<0.001$ \\
Importance of mothers' approval & 0.146 & 0.008 \\
Perceptions of mothers' attitudes towards teen sex with a steady boyfriend & 0.115 & 0.038 \\
Condom use self-efficacy & 0.100 & 0.068 \\
Intentions to have sex in next 3 months $\left(r_{s}\right)$ & -0.101 & 0.068 \\
\hline
\end{tabular}

Note: $N=330$. Daughters' baseline reports unless otherwise indicated. Pearson product-moment correlation coefficient $(r)$ unless otherwise indicated. ${ }^{\text {a } M o t h e r s ' ~ b a s e l i n e ~ r e p o r t s . ~}$

mothers' reports of PTSRC. Daughters' PTSRC scores were also associated with daughters' reports of comfort and ease with mother-daughter sexual communication, mother-daughter closeness, importance of mothers' approval, and daughters' perceptions that their mothers held more liberal attitudes towards teen sex with steady boyfriends. In addition, adolescent girls who reported greater amounts of PTSRC with mothers reported marginally greater condom use self-efficacy and marginally lower intentions to have sex in the next 3 months. Daughters' ages were not associated with daughters' PTSRC-III scores.

\section{DISCUSSION}

The current study findings demonstrated that the PTSRC-III scale performed well when used with urban Jamaican female adolescents and their mothers. Psychometric analyses found high levels of internal reliability, stability in reports across time, consistent two-factor structure, and associations with parent-child closeness, perceived importance of mothers' opinions, and perceptions of mothers' attitudes toward teen sex with a steady boyfriend. Marginal associations were also found between greater amounts of mother-daughter sexual communication and daughters' reports of increased condom use self-efficacy and fewer intentions to engage in sex.

The findings of the current study were consistent with reports in the published literature. Jamaican female adolescents' mean scores on sexual risk communication with their mothers were comparable to those reported for college students, late adolescent female drivers and African American college students in earlier US studies [24,31]. Mean PTSRC scores were 25.66 (9.14) in the current study, compared to 25.41 (8.55) for African American female late adolescent drivers [24], and 23.97 (9.43) for
African American female college students [31]. Congruence between mothers' and daughters' reports of sexual communication was found to be moderate, with mothers reporting greater amounts of sexual communication than daughters; these findings were also consistent with the published literature $[31,38,50]$.

The PTSRC-III demonstrated excellent internal reliability among Jamaican adolescent girls and mothers $(\alpha$ $=0.93$ and 0.92, respectively). Inter-item correlations were all strongly and positively correlated with one another. The high level of internal consistency was similar to that found when the PTSRC-III scale was used in earlier studies with adolescent females in the US ( $\alpha=0.93$ and 0.92) [24,31] and adolescents in Mexico $(\alpha=0.94)$ $[37,38]$. Similar levels of internal reliability were also found when the instrument was used in South Africa $(\alpha=$ 0.92) (Hutchinson, M.K., personal communication).

Stability of PTSRC-III scores over time was also demonstrated. The test-retest correlations over a 3-month period were moderate to large and statistically significant for both daughters $(r=0.57, p<0.001)$ and mothers $(r=$ $0.70, p<0.001)$. Although traditionally one aims for testretest reliability coefficients $>0.80$, the 3 -month timeframe between the two measurement points was long [47, 48]. More importantly, because communication is not a time-invariant trait, one would expect some variation across time. Parent-teen sexual communication would be expected to change, ebb and flow, as the adolescent grows and develops and her social situation, dating and/or sexual behaviors change. As the PTSRC scale questioned respondents about total amounts of informational sexual exchange, one would expect that scores would increase over time.

The factor structure for the PTSRC-III scale was the one area in which minor differences were found between 
the current study's findings in Jamaica and previous US studies. As was reported, the factor analysis found two factors. One factor loaded with an eigenvalue greater than 1.0. This factor had a very similar loading pattern, comparable eigenvalue and explanation of variance as in the initial psychometric testing of the PTSRC-III instrument [24]. Although the second factor in this study did not have an eigenvalue greater than 1.0; the loading pattern and explanation of variance were very similar to the factor 2 described in the initial psychometric testing of the PTSRC-III instrument [24]. As in the initial assessment of the instrument, the two factors were highly correlated $(r>0.65)$.

\section{CONCLUSION}

Instruments developed and tested in one culture may not produce dependable results when used in different cultures. The cross-cultural validity and reliability of instruments must be evaluated before researchers may be confident that the obtained scores are accurate and reliable representations of the construct in the new cultural context $[44,45,51]$. The PTSRC-III scale has been found to be both valid and reliable for use in Jamaica and able to produce consistent results across cultures. The crosscultural applicability of the instrument allows for international comparisons.

\section{ACKNOWLEDGEMENTS}

This research was funded by a grant from the National Institutes of Health/National Institute of Nursing Research (R01 NR010478; M.K. Hutchinson, PI).

\section{REFERENCES}

[1] UNAIDS (2010) UNAIDS report on the global AIDS epidemic. http://www.unaids.org/globalreport/

[2] Ministry of Health of Jamaica (2009) National HIV/STI program-Jamaica HIV epidemic update January to December 2009.

http://www.nhpjamaica.org/jamaica-hiv-epidemic-update -january-december-2009

[3] National HIV/STD Prevention \& Control Program (2004) HIV/AIDS epidemic. Update 2004.

http://www.jis.gov.jm/special_sections/aids/AIDSDATAJ UNE2004.pdf

[4] Ministry of Health of Jamaica (2008) National HIV program, Jamaica country progress report. http://www.jamaica-nap.org/UNGASSCountryProgressR eport.pdf

[5] Figueroa, J.P., Duncan, J., Byfield, L., Harvey, K., Gebre, Y., Hylton-Kong, T., Hammer, F., Williams, E., Carrington, D. and Braithwaite, A.R. (2008) A comprehensive response to the HIV/AIDS epidemic in Jamaica: A review of the past 20 years. West Indian Medical Journal, 57, 562-576.
[6] UNAIDS Jamaica (2010) HIV/aids health profile. http://www.usaid.gov/our work/global health/aids/Count ries/lac/jamaica_profile.pdf.

[7] Chevannes, B. (2001) Learning to be a man: Culture, socialization, and gender identity in five Caribbean communities. University of West Indies Press, Jamaica.

[8] Ekundayo, O.J., Dodson-Stallworth, J., Roofe, M., Aban, I.B., Bachmann, L.H., Kempf, M.M.C., Ehiri, J. and Jolly, P.E. (2007) The determinants of sexual intercourse before age16 years among rural Jamaican adolescents. Scientific World Journal, 7, 493-503. doi:10.1100/tsw.2007.94

[9] Olukoga, I.A. (2004) Epidemiologic trends of HIV/AIDS in Jamaica. Public Health, 15, 358-363.

[10] Rolfe, B., Hemmings, J., Morris, T.A. and Samuels-Dixon, V. (2007) She sweet up the boopsy and him nuh get nuh wine: Young women and sexual relationships in Kingston, Jamaica. http://carisma-pancap.org/attachments/143 FinalPeerJam aicaReport.pdf

[11] Smikle, M.F., Dowe., G, Hylton-Kong, T., Williams, E. and Baum, M. (2000) Risky behavior in Jamaican adolescents' patients attending a sexually transmitted disease clinic. West Indian Medical Journal, 49, 327-330.

[12] Baumgartner, J.N., Waszak, G.C., Tucker, H. and Wedderburn, M. (2009) The influence of early sexual debut and sexual violence on adolescent pregnancy: A matched case-control study in Jamaica International Perspectives on Sexual and Reproductive Health, 35, 21-28. doi:10.1363/3502109

[13] Jackson, J., Leitch, J., Lee, A., Eggleston E. and Hardee K. (1998) The Jamaica adolescent study.

http://www.fhi.org/NR/rdonlyres/eucyp6dqvxf2kqoj2lrys c36hez3c3fzmhx6lhkoszpmv6ayow37vgd222j6lx5rg3oh vhzhy4kr3l/Jamaica1f.pdf

[14] Thomas, T. (2006). Youth reproductive and sexual health in Jamaica.

http://www.advocatesforyouth.org/storage/advfy/docume nts/fsjamaica.pdf

[15] Wilks, R., Younger, N., McFarlane, S., Francis, D. and van den Broeck, V. (2006) Jamaica youth risk and resiliency behaviour survey 2006; a baseline community-based survey of youth aged 15 - 19 .

http://www.nhpjamaica.org/informationCentre/publications

[16] Hope Enterprise Limited (2008) HIV/AIDS knowledge attitudes and behavior survey.

http://www.moh.gov.jm/general/tender/doc details/47-kn owledge-attitude-a-behavior-survey-2008

[17] Wood, E., Hutchinson, M.K., Kahwa, E., Hewitt, H. and Waldron, N. (in press) Relationships between adolescent girls and older partners in Jamaica. Journal of Nursing Scholarship.

[18] DiClemente, R., Wingood, G., Crosby, R., Sionean, C., Cobb, B., Harrington, K., et al. (2002) Sexual risk behaviors associated with having older sex partners. Sexually Transmitted Diseases, 29, 20-24. doi:10.1097/00007435-200201000-00004

[19] Miller, K., Clark, L. and Moore, J. (1997) Sexual initiation with older male partners and subsequent HIV risk 
behavior among female adolescents. Family Planning Perspectives, 29, 212-214. doi:10.2307/2953397

[20] DiIorio, C., Kelleym, M. and Hockenberry-Eaton, M. (1999) Communication about sexual issues: Mothers, fathers, and friends. Journal of Adolescent Health, 24, 181-189. doi:10.1016/S1054-139X(98)00115-3

[21] Karofsky, P.S., Lan Zeng, M.S. and Kosorok, M.R. (2001) Relationship between adolescent-parental communication and initiation of first intercourse by adolescents. Journal of Adolescent Health, 28, 4-45. doi:10.1016/S1054-139X(00)00156-7

[22] Rose, A., Koo, H.P., Bhaskar, B., Anderson, K., White, G. and Jenkins, R.R. (2005) The influence of primary caregivers on the sexual behavior of early adolescents. Journal of Adolescent Health, 37, 135-144. doi:10.1016/j.jadohealth.2005.02.009

[23] Fasula, A.M. and Miller, K.S. (2006) African-American and Hispanic adolescents' intention to delay first intercourse: Parental communication as a buffer for sexually active peers. Journal of Adolescent Health, 38, 193-200. doi:10.1016/j.jadohealth.2004.12.009

[24] Hutchinson, M.K. (2007) The parent-teen sexual risk communication scale: Instrument development and psychometrics. Nursing Research, 56, 1-8. doi:10.1097/00006199-200701000-00001

[25] Pearson, J., Muller, C. and Frisco, M.L. (2006) Parental involvement, family structure, and adolescent sexual decision making. Sociological Perspective, 49, 67-90. doi:10.1525/sop.2006.49.1.67

[26] Dilorio, C., McCarty, F., Denzmore, P. and Landis, A. (2007) The moderating influences of mother-adolescent discussion on early and middle African American adolescent sexual behavior. Research in Nursing Health, 30, 193-202.doi:10.1002/nur.20193

[27] Eisenberg, M.E., Sieving, R.E., Bearinger, L.H., Swain, C. and Resnick, M.D. (2006) Parent communication with adolescents about sexual behavior: A missed opportunity for prevention? Journal of Youth Adolescence, 35, 893902. doi:10.1007/s10964-006-9093-y

[28] Whitaker, D.J. and Miller, K.S. (2000) Parent-adolescent discussions about sex and condoms: Impact on peer influences of sexual risk behavior. Journal of Adolescent Research, 15, 251-273. doi:10.1177/0743558400152004

[29] Hutchinson, M.K. (2002) The influence of sexual risk communication between parents and daughters on sexual risk behaviors. Family Relation, 51, 238-247. doi:10.1111/j.1741-3729.2002.00238.x

[30] Hutchinson, M.K., Jemmott, J.B., Jemmott, L.S., Braverman, P. and Fong, G.F. (2003) The role of mother-daughter sexual risk behaviors among urban adolescent females: A prospective study. Journal of Adolescent Health, 33, 98-107. doi:10.1016/S1054-139X(03)00183-6

[31] Hutchinson, M.K. and Montgomery, A.J. (2007) Parent communication and sexual risk among African Americans. Western Journal of Nursing Research, 29, 691-707. doi:10.1177/0193945906297374

[32] Guilamo-Ramos, V., Jaccard, J., Dittus, P. and Collins, S. (2008) Parent-adolescent communication about sexual intercourse: An analysis of maternal reluctance to communicate. Health Psychology, 27, 760-769. doi:10.1037/a0013833

[33] DiClemente, R. J., Wingood, G. M., Crosby, R., Sionean, C. M., Cobb, B. K., Harrington, K., Davies, S., Hook, E. W. and Oh, M. K. (2001) Parental monitoring: Association with adolescents' risk behaviors. Pediatrics, 107, 13631368. doi:10.1542/peds.107.6.1363

[34] Longmore, M., Manning, W. and Giordano, P. (2001) Preadolescent parenting strategies and teens' dating and sexual initiation: A longitudinal analysis. Journal of Marriage and the Family, 63, 233-335. doi:10.1111/j.1741-3737.2001.00322.x

[35] Hutchinson, M.K. and Wood, E. (2007) Reconceptualizing adolescent sexual risk using a parent-based expansion of the Theory of Planned Behavior. Journal of Nursing Scholarship, 39, 141-146. doi:10.1111/j.1547-5069.2007.00159.x

[36] Pequegnat, W. and Szapocznik, J. (2000) The role of families in preventing and adapting to HIV/AIDS: Issues and answers. In: Pequegnat, W. and Szapocznik, J. Eds., Working with Families in the Era of HIV/AIDS, Sage, Thousand Oaks, 3-26.

[37] Villarruel, A.M., Loveland Cherry, C.L., Cabriales, E.G., Ronis, D.L. and Zhou, Y. (2008) A parent-adolescent intervention to Increase sexual risk communication: Results of a randomized controlled trial. AIDS Education and Prevention, 20, 371-383. doi:10.1521/aeap.2008.20.5.371

[38] Gallegos, E., Villarruel, A., Vinicio Gomez, M., Onofre, D. and Zhou, Y. (2007) Sexual communication and knowledge among Mexican parents and their adolescent children. Journal of the Association of Nurses in AIDS Care, 18, 28-34. doi:10.1016/j.jana.2007.01.007

[39] Jaccard, J. and Dittus, P. (1991) Parent-teen communication: Toward the prevention of unintended pregnancies. Springer-Verlag, New York.

[40] Lenciauskiene, I. and Zaborskis, A. (2008) The effects of family structure, parent-child relationship and parental monitoring on early sexual behavior among adolescents in nine European countries. Scandinavian Journal of Public Health, 36, 607-618. doi:10.1177/1403494807088460

[41] Ajzen, I. (1991) The theory of planned behavior. Organizational Behavior and Human Decision Processes, 50, 179-211. doi:10.1016/0749-5978(91)90020-T

[42] Jemmott, L.S., Jemmott, J.B., and Hutchinson, M.K. (2001) HIV/AIDS: Prevention needs and strategies for a public health emergency. In: R. Braithwaite Ed., Health Issues in the Black Community (2nd Edition), Jossey-Bass, San Francisco, 309-346.

[43] Hutchinson, M. K., Jemmott, L. S., Wood, E., Hewitt, H., Kahwa, E., Waldron, N. and Bonaparte, B. (2007) Culture-specific factors contributing to HIV risk among Jamaican adolescents. Journal of the Association of Nurses in AIDS Care, 18, 35-47. doi:10.1016/j.jana.2007.01.008

[44] Van de Vijver, F.J.R. and Poortinga, Y.H. (1997) Towards an integrated analysis of bias in cross-cultural assessment. European Journal of Psychological Assessment, 13, 29- 


\section{7. doi:10.1027/1015-5759.13.1.29}

[45] Van de Vijver, F. and Tanzer, N.K. (2004) Bias and equivalence in cross-cultural assessment: An overview. Revue Européenne de Psychologie Appliquée, 54, 119-135.

[46] Hutchinson, M.K., Hewitt, H., Waldron, N., Kahwa, E., Hamilton, P., Jemmott, L. and Jemmott, J. (2011) Development and testing of a Jamaican mother-daughter HIV risk-reduction intervention. National Institute of Nursing Research 25th Anniversary Research Poster Session, Washington DC, 13 October 2011.

[47] Burns, N. and Grove, S. (2009) The practice of nursing research: Appraisal, synthesis and generation of evidence. 6th Edition, Saunders Elsevier, St. Louis.
[48] Nunnally, J.C. (1978) Psychometric theory. 2nd Edition, McGraw-Hill, New York.

[49] Pett, M.A., Lackey, N.R. and Sullivan, J.J. (2003) Making sense of factor analysis: The use of factor analysis for instrument development in health care research. Sage, Thousand Oaks.

[50] Jaccard, J., Dittus, P. and Gordon, V. (1998) Parent-adolescent congruency in reports of adolescent sexual behavior and in communications about sexual behavior. Child Development, 69, 247-261.

[51] Aghanwa, H.S., Walkey, F.H. and Taylor, A.J.W. (2003) The psychometric cross-cultural validation of the impact of event scale. Pacific Health Dialog, 10, 66-70. 